\title{
Investigating the Adsorption Behavior and Mechanism of Eu(III) and Au(III) on $\beta$ - cyclodextrin/polyethylenimine Functionalized Waste Paper
}

\section{Fenglei Liu}

Shaoxing University

Shan Hua

Shaoxing University

Qingyuan Hu

Shaoxing University

Chao Wang

Shaoxing University

baowei hu ( $\square$ hbw@usx.edu.cn )

Shaoxing University

\section{Research Article}

Keywords: Waste paper, $\beta-\mathrm{CD}, \mathrm{Eu}(\mathrm{III}), \mathrm{Au}(\mathrm{III})$, Adsorption

Posted Date: September 21st, 2021

DOI: https://doi.org/10.21203/rs.3.rs-892094/v1

License: (a) (i) This work is licensed under a Creative Commons Attribution 4.0 International License. Read Full License

Version of Record: A version of this preprint was published at Cellulose on January 29th, 2022. See the published version at https://doi.org/10.1007/s10570-021-04389-2. 


\section{Abstract}

A bio-adsorbent (DAWP-PEI- $\beta$-CD) was facilely prepared by introducing polyethylenimine (PEI) and $\beta$ cyclodextrin ( $\beta-C D)$ into dialdehyde waste paper (DAWP) via a facile two-step method. Various physicochemical and spectroscopic techniques (FT-IR, XRD, SEM, NMR, XPS) were applied to characterize the structure, morphology and composition of the as-prepared adsorbents. Investigation results showed that the $\mathrm{pH}$ values, reaction temperature and contact time played a vital role in uptake of $\mathrm{Eu}(\mathrm{III})$ and $\mathrm{Au}(\mathrm{III})$. Meanwhile, the adsorption behavior of $\mathrm{Eu}(\mathrm{III})$ and $\mathrm{Au}$ (III) could be fitted felicitously with the Langmuir and the Pseudo-second-order models, and the saturated adsorption amounts of Eu(III) $(\mathrm{pH}=$ 6.0) and $\mathrm{Au}(\mathrm{III})(\mathrm{pH}=2.0)$ onto DAWP-PEI- $\beta-C D$ were 424.2 and $241.3 \mathrm{mg} / \mathrm{g}$, respectively. Further advanced spectroscopy analysis revealed that the elimination of Eu(III) was attributed to host-guest inclusion and surface complexation interaction, while adsorption of $\mathrm{Au}$ (III) might stem from a combination of electrostatic attraction, chelation, host-guest inclusion and redox interaction. This study demonstrated that DAWP-PEI- $\beta$-CD was a promising environmental functional material to separation and enrichment of $\mathrm{Eu}(\mathrm{III})$ and $\mathrm{Au}(\mathrm{III})$ from contaminated water.

\section{Introduction}

With the rapid promotion of urbanization, the radionuclides and precious metals pollution have become an imperative global environment problem, especially for europium (Eu) and Aurum (Au) (Zhao et al., 2015). Generally, europium has major applications in neutron protection, laser and atomic energy industry field, while gold is widely used in the fields of industry, agriculture and medicine (Neri et al., 2021, Zhao et al., 2021). However, a large amount of wastewater containing $\mathrm{Eu}(\mathrm{III})$ or $\mathrm{Au}(\mathrm{III})$ were produced in the process of application. Thereby, how to efficiently elimination of Eu(III) and Au(III) from the polluted water sources or industrial wastes to avoid these pollutants migration and transformation is a very pressing issue. In an effort to handle those challenges, several state-of-the-art techniques have been proposed to purify $\mathrm{Eu}$ (III) or $\mathrm{Au}$ (III)-containing wastewater, such as chemical precipitation (Yan et al., 2019), membrane processing (Wang et al., 2020), electrolysis (Liu et al., 2019) and adsorption (Zhang et al., 2019). Amount them, adsorption seems to be one of the most effective and practical technologies owing to its simplicity and flexibility feature. Nowadays, different types of adsorbents have been developed and evaluated for Eu and Au ions clean-up, such as carbon nanotubes (Chen et al., 2008), graphene oxide (Ma et al., 2019), viscose fiber (Liu et al., 2020) and corn starch (Liu et al., 2019). However, these solid materials generally have the defects of low adsorption amounts, long equilibrium time and weak stability under various environmental conditions. One suggested approach is to the preparation of new materials with high adsorption capacity and high chemical stability, so that it can be used to efficient elimination of the two contaminant classes.

Recently, biomass materials have stimulated researchers' interest on account of their renewable, free of contamination and low cost characteristics (Zhu et al., 2021). So far, different types of bio-materials have been applied to eliminate the radionuclide and precious metals ions from the wastewater stream, such as tannin (Liu et al., 2021), chitosan (Liu et al., 2021) and cellulose (Zhang et al., 2021). Indeed, $\beta$ - 
cyclodextrin ( $\beta-C D)$ is also an inexpensive sustainable biomass resource, which is mainly produced from the enzymatic degradation of starch. More importantly, $\beta-C D$ is cyclic oligosaccharides formed by $6-8 D$ glucose units linked by a-1,4-glucose bonds, which has been proved to exhibit good performance in wastewater disposal (Ghosh et al., 2011). However, $\beta$-CD requires a process of immobilization/insolubilization before being used as adsorbents for aqueous phase applications due to its water solubility. To compensate for this disadvantage, many efforts have been made to immobilize $\beta$ CD onto various water-insoluble matrices. For instance, Zhao et al. (Zhao et al., 2016) developed an EDTA- $\beta$-cyclodextrin material to pre-concentration of Eu(III) from simulated seawater, and the maximum adsorption capacities reached up to $0.365 \mathrm{mmol} / \mathrm{g}$. Martin-Trasanco et al. (Martin-Trasanco et al., 2017) reported a $\beta$-cyclodextrin polymer to selective recovery of $\mathrm{Au}$ (III) from acid medium, and it was observed that $\mathrm{Au}$ (III) ions could be efficient reduced to gold nano-particles by hydroxys groups. Guo et al. (Guo et al., 2015) synthesized a $\mathrm{Fe}_{3} \mathrm{O}_{4} @$ @yclodextrin magnetic composite via a simple chemical co-precipitation method, and this magnetic composites could efficient removal of Eu(III) from industrial wastewater. Kyrychenko et al. (Slavgorodska et al., 2020) explored the adsorption behavior of $\beta$-cyclodextrin onto gold nano-particles, and it was found that $\beta$-CD binding onto the AuNP surface occurred through multiple noncovalent interactions. However, Although these surface modification strategies could effective immobilize $\beta$-CD on insoluble carrier, most of these methods use complicated procedures and toxic reagents, and the majority of the previously reported $\beta-C D$ based adsorbents suffered from the low adsorption amounts and slow reaction rates toward the targeted contaminants. Consequently, there is an urgent need to propose a new $\beta-C D$ immobilization strategy to elimination of the targeted pollutants. It is worth noting that waste paper (WP) is an inexpensive, renewable and massively available material, of which the main components are cellulose and hemicellulose. Notably, WP is easy to be oxidized by strong oxidant, which will produce a large amount of aldehyde groups on its skeleton. Furthermore, the aldehyde groups could form covalent and hydrogen bonds with the amine and hydroxy functional groups under the mild environmental conditions. Polyethylenimine a kind of sterically branched polymer with larger numbers of amino groups, such as primary, secondary and tertiary amine groups. And one third of the atoms of PEI are protonatable amino nitrogen atoms, which exhibits high affinity towards metal ions (Zhao et al., 2017). Hence, the special chemical structure of DAWP opened up an opportunity for the facile introduction of $\beta-C D$ and $P E I$ through one step or two-step synthetic strategies.

The purposes of this study were to: (1) to develop a novel bio-adsorbent (DAWP-PEI- $\beta$-CD) via a facile two-step method and investigate its adsorption performance towards $\mathrm{Eu}(\mathrm{III})$ and $\mathrm{Au}$ (III) under various environmental conditions; (2) to characterize the as-synthesized bio-adsorbents by different physicochemical and spectroscopic techniques (FT-IR, XRD, SEM, NMR, XPS); (3) to evaluate the DAWPPEI- $\beta$-CD performance for $\mathrm{Eu}(\mathrm{III})$ and $\mathrm{Au}(\mathrm{III})$ uptake using the kinetic and isotherm models; (4) to understanding the interaction mechanism between DAWP-PEI- $\beta-C D$ and $\mathrm{Eu}(\mathrm{III}) / \mathrm{Au}(\mathrm{III})$ ions. This study will provide new clues to the overall recycling of $\mathrm{Eu}(\mathrm{III})$ and $\mathrm{Au}(\mathrm{III})$ in environmental pollution treatments.

\section{Materials And Methods}




\section{Materials}

$\beta$-Cyclodextrin ( $\beta-C D, 98 \%$ ), polyethyleneimine (PEI, M.W.600, 99\%), epichlorohydrin (EPI, AR), $\mathrm{Eu}\left(\mathrm{NO}_{3}\right)_{3} \cdot 6 \mathrm{H}_{2} \mathrm{O}(99.9 \%)$ and $\mathrm{HAuCl}_{4} \cdot 4 \mathrm{H}_{2} \mathrm{O}(99.9 \%)$ were purchased from Sinopharm Reagent Co. Ltd., China. The $\mathrm{NaCl}$ (99.5\%), $\mathrm{FeCl}_{2}$ (98\%), $\mathrm{NaIO}_{4}$ (99.5\%), $\mathrm{NaOH}(95 \%), \mathrm{HCl}(37 \%), \mathrm{CuCl}_{2}(98 \%), \mathrm{ZnCl}_{2} .7 \mathrm{H}_{2} \mathrm{O}$ (98\%), $\mathrm{NiCl}_{2} \cdot 6 \mathrm{H}_{2} \mathrm{O}(99.9 \%), \mathrm{FeCl}_{3} \cdot 6 \mathrm{H}_{2} \mathrm{O}(99 \%)$ and $\mathrm{K}_{2} \mathrm{Cr}_{2} \mathrm{O}_{7}$ (98\%) were received by Shanghai Macklin Biochemical Co., Ltd. Waste paper (WP) was obtained from personal laboratory and crushed into small pieces mechanically before use.

\section{Preparation of DAWP-PEI- $\beta-C D$}

The preparation process of bio-adsorbent (DAWP-PEI- $\beta-C D$ ) was proposed as Fig. 1. Firstly, $2 \mathrm{~g}$ of waste paper was firstly added into $60 \mathrm{~mL}$ of a sodium periodate solution $(\mathrm{pH}=4.0,0.7 \mathrm{~mol} / \mathrm{L})$. Then, the mixtures were stirred at $313 \mathrm{~K}$ for $5 \mathrm{~h}$ under exclusion of light, and the oxidized waste papers were obtained and named as DAWP. Secondly, $1 \mathrm{~g}$ of DAWP was added into $50 \mathrm{~mL}$ of PEl solution (5 wt.\%) to form a suspension, which was continuous stirred at room temperature for $12 \mathrm{~h}$ to acquired yellow solids (DAWP-PEI). Finally, $4.8 \mathrm{~g}$ of EPI and $10 \mathrm{~g}$ of $\beta$-CD were dissolved into $100 \mathrm{~mL}$ of $\mathrm{NaOH}$ solution (7 wt.\%). And then $2 \mathrm{~g}$ of DAWP-PEI was joined with the modification solution under intense stirring for $6 \mathrm{~h}$. After that, the mixture was filtered with ultra-pure water to make it neutral and the as obtained bio-adsorbents (DAWP-PEI- $\beta-C D$ ) were dried at in an oven for further use.

\section{Characterization}

The Fourier transform infrared spectrometer (FT-IR) spectra were analyzed on the NEXUS 870 spectroscopy (Thermo, Madison, USA). X-ray photoelectron spectroscopy (XPS) data were registered in an ESCALAB 250 XPS (ThermoFisher, AIKa). The morphological analysis was carried out by scanning electron microscope (SEM) using a QUANTAFEG 400 (FEl company, USA), and elemental mapping was performed by Thermo Scientific Ultra Dry SDD Energy-dispersive X-ray spectroscopy (EDS, USA). The Xray diffraction (XRD) analysis was performed on a Rigaku TTRIII diffractometer (Japan) with Cu Ka irradiation over a $2 \theta$ range from 20 to $80^{\circ} .{ }^{13} \mathrm{C}$ Solid state nuclear magnetic spectrum (NMR) were recorded on a Bruker AVANCE II 400WB spectrometer (Germany). Surface charge density of samples was determined by a Malvern Instruments Zetasizer Nano ZS90 (England).

\section{Batch removal experiments}

Batch experiments were carried out to explore the capture performance of $\mathrm{Eu}(\mathrm{III})$ and $\mathrm{Au}(\mathrm{III})$ on DAWP-PEI$\beta$-CD. Here, $10 \mathrm{mg}$ of DAWP-PEI- $\beta$-CD was added into $50 \mathrm{~mL}$ of known concentration $\mathrm{Eu}$ (III) or $\mathrm{Au}$ (III) solution with the desired $\mathrm{pH}$ values. Then, the suspensions were shaken in the incubator at $250 \mathrm{rpm}$ for $24 \mathrm{~h}$ until adsorption equilibrium. Upon the completion, the solid adsorbents were separated from solution phase by centrifugation. Subsequently, the supernatant concentration of Eu(III) was analyzed by the UV-vis adsorption spectrometer at the wavelengths $652 \mathrm{~nm}$, whereas the residual concentration of $\mathrm{Au}(\mathrm{III})$ was conformed by ICP-AES. The adsorption ability of DAWP-PEI- $\beta$-CD was performed by transferring $10 \mathrm{mg}$ of bio-adsorbents into $50 \mathrm{~mL}$ of targeted pollutants, which already contained a series 
of different concentrations of $\mathrm{Eu}(\mathrm{III})$ and $\mathrm{Au}$ (III) solution. The kinetic experiment of $\mathrm{Au}(\mathrm{III})$ and $\mathrm{Eu}$ (III) on DAWP-PEI- $\beta$-CD were carried out at optimal $\mathrm{pH}$ values, and the concentrations of metal ions were measured and recorded at different time points until adsorption equilibrium. The adsorbed amounts of $\mathrm{Eu}(\mathrm{III})$ and $\mathrm{Au}(\mathrm{III})$ onto DAWP-PEI- $\beta$-CD were calculated from the difference of initial concentration and final concentration remained in solution after equilibrium.

\section{Results And Discussion}

\section{Adsorbent characterization}

XPS analyses were used to investigate the surface chemical compositions of DAWP, DAWP-PEI and DAWP-PEI- $\beta$-CD. As showed in Fig. 2 , the $C 1 \mathrm{~s}$ spectrum of DAWP could be quantitatively resolved into three bonds at 284.81, 286.62 and $288.12 \mathrm{eV}$ corresponded to carbon-carbon bond (C-C), carbon-oxygen bond $(\mathrm{C}-\mathrm{OH})$ and carbon and oxygen double bond $(\mathrm{C}=0)$, respectively. Interestingly, once the PEI molecules were immobilized on DAWP via Schiff base reaction, two new peaks appeared at $287.15 \mathrm{eV}$ (C $=\mathrm{N}$ ) and $285.74 \mathrm{eV}(\mathrm{C}-\mathrm{N})$, proving that a mass of amino groups existed on the surface of DAWP-PEI (Liu et al., 2020). However, as the $\beta$-CD molecules were further introduced into DAWP-PEl through cross-linking reaction, the relative content of C-N on DAWP-PEI (14.20\%) was lower than that of DAWP-PEI- $\beta$-CD (25.58\%), and other characteristic peaks were still presented in DAWP-PEI- $\beta$-CD. These observations conformed that PEI and $\beta-C D$ molecules had been successfully immobilized on DAWP via a facile twostep method.

FT-IR measurements were mostly used in order to confirm the formation of functional groups. Thus, different functional groups were detected in the IR spectra of $\beta-C D$, DAWP, DAWP-PEI and DAWP-PEI- $\beta-C D$. In Fig. $2 d$, the typical peaks of $\beta-\mathrm{CD}$ at $3352 \mathrm{~cm}^{-1}$ and $1208 \mathrm{~cm}^{-1}$ were generated by the $-\mathrm{OH}$ and $-\mathrm{CH}_{2}$ bending vibrations, whereas another band at $1059 \mathrm{~cm}^{-1}$ was produced by inverse-symmetric glycosidic (Wang et al., 2014). The bands centered at $1688 \mathrm{~cm}^{-1}, 1427 \mathrm{~cm}^{-1}$ and $1109 \mathrm{~cm}^{-1}$ ascribed to $-\mathrm{C}=0,-\mathrm{CH}_{2}$ and C-O-C stretching vibrations of DAWP, respectively (Liu et al., 2019). Notably, once the PEI molecules were introduced into oxidized waste paper, two regular peaks appeared at $1649 \mathrm{~cm}^{-1}$ and $1422 \mathrm{~cm}^{-1}$ corresponded to $-\mathrm{C}=\mathrm{N}$ and $-\mathrm{NH}_{2}$, respectively (Al-Harahsheha et al., 2020). Interestingly, after the crosslinking reaction, the characteristic peaks of $\beta-C D$ and $P E I$ were also appeared at the IR spectra of DAWPPEI- $\beta-C D$, such as primary amine $\left(1455 \mathrm{~cm}^{-1}\right)$ and the $b(1-4)$ skeleton vibrations $\left(1033 \mathrm{~cm}^{-1}\right)$. These analysis results further proved that the $\beta$-CD and PEI were successfully introduced into the oxidized waste paper.

The chemical structure of all adsorbents was further assessed by solid state NMR measurements. In Fig. 3 , one can see that the broad signals from $102 \mathrm{ppm}$ to $59 \mathrm{ppm}$ were attributed to the carbon atoms in $\beta$ $C D$, and the signals at $\delta=63,72,84$ and 102 ppm corresponding to C-d, C-a, C-e and C-b, respectively (Shen et al., 2015). Compared with $\beta-C D$, the characteristic peak of carbon atoms at $\delta=72 \mathrm{ppm}(C-2)$ and 65 ppm (C-4) were almost overlapped or disappeared in the NMR spectrum of DAWP, which may be 
related to the signal amplification of C-5 and C-3 after oxidation reaction. However, when DAWP was reacted with $\mathrm{PEI}$, there were several new characteristic peaks appeared at NMR spectra of DAWP-PEI. For instance, the carbon and nitrogen double bond $(\mathrm{C}=\mathrm{N})$ as well as carbon and nitrogen single bond $(\mathrm{C}-\mathrm{N})$ were observed at $\delta=143 \mathrm{ppm}$ and $30 \mathrm{ppm}$, respectively. Interestingly, most of DAWP and PEl's carbon atom signals were also detected on the NMR spectra of DAWP-PEI- $\beta$-CD after cross-linking reaction, and a different signal appeared at $\delta 169 \mathrm{ppm}$ may ascribed to $C=0$. These results were in accordance with FTIR and XPS results, which further manifested that the successful cross-linking between DAWP and $\beta$ $\mathrm{CD} / \mathrm{PEI}$.

\section{Influences of $\mathrm{pH}$ values}

As is well-known, solution $\mathrm{pH}$ not only can affect the relative distribution of pollutants species but also change the surface charge of adsorbents. Thus, the adsorption abilities of DAWP-PEI- $\beta$-CD towards Eu(III) and $\mathrm{Au}$ (III) were investigated in this work. As depicted in Fig. 3a, adsorption amounts of Eu(III) on DAWPPEI- $\beta$-CD were up to $282 \mathrm{mg} / \mathrm{g}$ rapidly from 2.0 to 5.0 , subsequently maintained the high level within pH the range of $6.0-9.0$, which implied that solution $\mathrm{pH}$ played a vital role in elimination of contaminants. This phenomenon could be explained by the relative distribution of Eu(III) species and the surface charge of DAWP-PEI- $\beta$-CD. It was reported that Eu(III) ions were mainly presented as $\mathrm{Eu}^{3+}$ species at $\mathrm{pH}<5.0$ in water solution, and then $\mathrm{Eu}(\mathrm{OH})^{2+}$ and $\mathrm{Eu}_{2}(\mathrm{OH})_{2}{ }^{4+}$ species began to increase with $\mathrm{pH}$ further increasing (Li et al., 2020). Meanwhile, zeta potential analyzer determined the surface charge density of DAWP-PEI- $\beta$ CD was 3.29 (Fig. S1). Thus, according to Coulomb's law, the positive charge of Eu(III) species were more easily adsorbed on the negatively charged DAWP-PEI- $\beta$-CD through electrostatic attraction. However, the adsorption behavior of $\mathrm{Au}(\mathrm{III})$ was significantly different from that of $\mathrm{Eu}(\mathrm{III})$ at various $\mathrm{pH}$ values. In Fig. $3 \mathrm{~b}$, one can see that adsorbed amounts of $\mathrm{Au}(\mathrm{III})$ on DAWP-PEI- $\beta$-CD increased sharply as pH values were raised from 1.0 to 2.0, and then sharply decreased over the $\mathrm{pH}$ range 2.0-6.0, and achieving its best performance at $\mathrm{pH}$ 2.0. Such a pH-dependent adsorption can be explained from the point of view of the following aspects. At lower $\mathrm{pH}$ values, the $\mathrm{Au}(\mathrm{III})$ ions were mainly existed as $\mathrm{AuCl}_{4}{ }^{-}$, which were favorable to capture on the positively-charged DAWP-PEI- $\beta$-CD via electrostatic interactions. Notably, the chloride ion occupied a part of the active sites during adsorption process at $\mathrm{pH}<2.0$, which led to a lower adsorption amounts (Wang et al., 2015). However, under high pH conditions, the negative charge increased on the surface of DAWP-PEI- $\beta$-CD and the electrostatic repulsion would occur between the bioadsorbents and adsorbates at high $\mathrm{pH}$ values.

\section{Adsorption isotherms and thermodynamic studies}

The adsorption isotherms are an important factor in understanding the adsorption efficiency and studying the adsorption mechanism. Figure 5 displays the relationship between the residual Eu(III) and $\mathrm{Au}$ (III) concentrations and the equilibrium adsorption capacities on DAWP-PEI- $\beta-C D$ at room temperature. Evidently, along with the initial concentration increasing, the adsorption amounts of $\mathrm{Eu}(\mathrm{III})$ and $\mathrm{Au}(\mathrm{III})$ on DAWP-PEI- $\beta$-CD rapidly increased at the beginning and then tended to adsorption equilibrium. Interestingly, the equilibrium adsorption capacities of $\mathrm{Au}$ (III) were both higher than that of Eu(III) on DAWP-PEI and DAWP-PEI- $\beta-C D$, which was evident that the greater affinity to $\mathrm{Au}$ (III) than $\mathrm{Eu}(\mathrm{III})$ in the 
adsorption process. To determine the saturated capture amounts and sorption type, the Langmuir and Freundlich equations were applied to fit the experimental data, and the detailed fitting results were provided in Fig. 5 and Table S1. As expected, the results of calculation and simulation showed that the adsorption behavior of $\mathrm{Eu}(\mathrm{III})$ and $\mathrm{Au}(\mathrm{III})$ were fitted well by the Langmuir model, which indicated that uptake of the two targeted contaminants onto DAWP-PEI- $\beta$-CD surfaces was localized in a monolayer (Yang et al., 2019). Besides, the adsorption thermodynamics of Eu(III) and Au(III) on DAWP-PEI- $\beta-C D$ were also measured using the Gibbs equations. As listed in Table S2, the spontaneous and endothermic adsorption processes were revealed by the negative $\Delta G^{\circ}$ and positive $\Delta H^{\circ}$ values, whereas the negative $\Delta S^{\circ}$ reflected a decrease in the randomness during the adsorption process. Notably, the maximum uptake amounts of DAWP-PEI- $\beta$-CD for single Eu(III) and Au(III) were 241.3 and $424.2 \mathrm{mg} \mathrm{mg} / \mathrm{g}$, which were far higher than that of the reported available materials (Table S3). Such a high adsorption amount could be ascribed to the special chemical structure and the large cavities, which were advantageous for accommodating more $\mathrm{Eu}(\mathrm{III})$ and $\mathrm{Au}(\mathrm{III})$.

\section{Adsorption kinetics}

Adsorption kinetics not only focuses on the relationship between adsorption capacity and adsorption time, but also evaluate the rate of the interaction process. Figure 6 illustrates the time-dependent experiments of $\mathrm{Eu}(\mathrm{III})$ and $\mathrm{Au}(\mathrm{III})$ adsorbed onto DAWP-PEI- $\beta$-CD. As excepted, the concentration of targeted contaminants rapidly declined during the initial phase, and then tend to a constant value with the extension of incubation time. The rapid adsorption in the beginning may be due to the greater concentration gradient and more available sites for adsorption. Subsequently, the active sites were gradually depleted, resulting in the final adsorption equilibrium. Note that the Au(III) exhibited faster kinetics compared with Eu(III) at optimal water environmental condition, which suggested that $\mathrm{Eu}(\mathrm{III})$ ions took a relatively longer time to diffuse into the interlayer of DAWP-PEI- $\beta$-CD. To figure out the adsorption rate and rate determining step, different kinetic models were applied to simulate the kinetic data, and the corresponding fitting curves and parameters were displayed in Fig. 6 and Table S4. According to the fitted results, on can see that the PFO equation either overestimated or underestimated the kinetics behavior of $\mathrm{Eu}(\mathrm{III})$ and $\mathrm{Au}$ (III) on DAWP-PEI- $\beta$-CD, while the PSO model could well simulate the kinetic process from the beginning to the end, and the calculated limits of quantification were close to the experimental limits of quantification, which implied that uptake of the two targeted pollutants mainly depended on chemical adsorption (Cheng et al., 2018, Wang et al., 2019). To further identify the main rate-controlling step, the kinetic data were further analyzed by the Intraparticle diffusion equation, and the plots of qt vs. $t^{1 / 2}$ were displayed in Fig. $6 \mathrm{c}$, d. The simulation results exhibited that the lines do not pass through the origin and tend to be multilinear, which manifested that the rate controlling steps did not only include intraparticle diffusion, and other reactions according to the definition of the equation (Qiu et al., 2020, Hu et al., 2021).

\section{Selectivity test}

Considering the practical application of the targeted pollutants removal from industrial wastewater, the effect of competing ions of adsorbents were also important. Thus, the selective binding ability of DAWPPEI- $\beta$-CD towards $\mathrm{Eu}(\mathrm{III})$ and $\mathrm{Au}(\mathrm{III})$ was investigated in a mixed system, in which the concentration of 
metal ions were all controlled at $50 \mathrm{mg} / \mathrm{L}$. In Fig. S2, one can see that the adsorption capacity of $\mathrm{Au}(\mathrm{III})$ on DAWP-PEI- $\beta$-CD was calculated as $209 \mathrm{mg} / \mathrm{g}$ in single system, and that was reduced by $20.1 \%$ in mixed system. This phenomenon occurred due to most of co-existing metal ions existed as their cationic or neutral forms, while $\mathrm{Au}$ (III) ions were mainly existed as $\mathrm{AuCl}_{4}{ }^{-}$in acidic solution. Thus, the negatively charged $\mathrm{Au}$ (III) species could occupy more adsorption sites on the surface of DAWP-PEI- $\beta$-CD, which led to a high $\mathrm{Au}(\mathrm{III})$ adsorption amounts. Besides, it should be pointed out that the molecular structure of $\mathrm{Eu}(\mathrm{III})$ were similar to that of $\mathrm{Au}$ (III) to some extent, but the removal amounts of $\mathrm{Eu}(\mathrm{III})$ was still lower than that of $\mathrm{Au}(\mathrm{III})$ at this adsorption conditions. This may be due to the electrostatic repulsion occurred between $\mathrm{Eu}(\mathrm{III})$ ions during adsorption process. This result manifested that DAWP-PEI- $\beta$-CD exhibited high selectivity for $\mathrm{Au}(\mathrm{III})$ against other co-existing pollutants in the multi-solute system.

\section{Elimination mechanisms}

To find out the detailed elimination mechanism, the as-prepared bio-adsorbent's surface properties and specific elemental constitutes were explored by SEM, XPS and XRD after binding with $\mathrm{Eu}(\mathrm{III})$ and $\mathrm{Au}(\mathrm{III})$. In Fig. 7, one can see that the main components of DAWP-PEI- $\beta$-CD were $C, H, N$ and $O$, whereas the element Eu and Au were spread over the whole surface of DAWP-PEI- $\beta$-CD-Eu and DAWP-PEI- $\beta$-CD-Au, suggesting that DAWP-PEI- $\beta$-CD could efficient enrichment of $E u(I I I)$ and $A u(I I I)$ from water environment. Interestingly, in Fig. $8 \mathrm{a}$, in addition to $\mathrm{C} 1 \mathrm{~s}$ and $01 \mathrm{~s}$, the spent DAWP-PEI- $\beta$-CD's surface also appeared characteristic peaks of Eu3d and Au4f, which was consistent with the results analyzed in EDS, further proved that the successful immobilization of the two targeted pollutants onto DAWP-PEI- $\beta$-CD.

To resolve the chemical properties and states of Eu and Au atoms on the DAWP-PEI- $\beta-C D$, the highresolution spectra of Eu3d and Au4f were further resolved by deconvolution method. In Fig. 8b, one could see that two main peaks located at $1134.49 \mathrm{eV}\left(\mathrm{Eu3d}_{3 / 2}\right)$ and $1164.16 \mathrm{eV}\left(\mathrm{Eu3d}_{5 / 2}\right)$ in the high-resolution Eu3d spectrum corresponded to the Eu(III) signals, which implied that only Eu(III) ions existed on the surface of DAWP-PEI- $\beta-C D$, and the surface complexes reaction dominated the processes of Eu(III) uptake on DAWP-PEI- $\beta$-CD (Huang et al., 2018). However, In Fig. 8c, the Au4f peak was mainly consisted of $\mathrm{Au}_{4 / 2}$ and $A u 4 f_{5 / 2}$, each of which could be further divided into $\mathrm{Au}(\mathrm{III})$ and $\mathrm{Au}(0)$. Specifically, the peaks at $83.36 \mathrm{eV}\left(\mathrm{Au}_{4 / 2}\right)$ and $87.08 \mathrm{eV}\left(\mathrm{Au}_{\left.7 \mathrm{f}_{5 / 2}\right)}\right.$ corresponded to $\mathrm{Au}(\mathrm{III})$, whereas the signatures at $84.18 \mathrm{eV}$ $\left(A u 4 f_{7 / 2}\right)$ and $89.05 \mathrm{eV}\left(\mathrm{Au}_{4 / 2}\right)$ were ascribed to $\mathrm{Au}(0)$, which demonstrated that a large amount of $\mathrm{Au}(0)$ existed onto the surface of spent bio-adsorbents (Pestov et al., 2015). This result was further proved by the XRD analysis. In Fig. $8 \mathrm{e}$, one can see that the powder XRD patterns of DAWP-PEI- $\beta$-CD exhibited two major peaks at $2 \theta=12.3^{\circ}$ and $18.1^{\circ}$, which may be ascribed to the characteristic peak of $\beta$ $\mathrm{CD}$. However, after adsorption of $\mathrm{Au}(\mathrm{III})$, there were four the high intensity peaks appeared at the XRD patterns of DAWP-PEI- $\beta$-CD-Au, and the reflections of elemental gold were observed at $2 \theta=38.19^{\circ}, 44.38^{\circ}$, $64.71^{\circ}$ and $77.52^{\circ}$ with the corresponding planes of (111), (200), (220) and (311). Noticeably, the specific area percentage of $\mathrm{C}=0$ enhanced from $12.09 \%$ to $15.78 \%$, and the content of $\mathrm{Au}(0)$ reached up to $54.65 \%$ after adsorption of $\mathrm{Au}(\mathrm{III})$ (see Table S5). These phenomena illustrated that part of surface adsorbed $\mathrm{Au}(\mathrm{III})$ has been reduced to $\mathrm{Au}(0)$ during the $\mathrm{Au}(\mathrm{III})$ capture processes. Besides, in Fig. 8f, the N1s peaks shifted from $398.86 \mathrm{eV}$ to $399.09 \mathrm{eV}$ after $\mathrm{Au}(\mathrm{III})$ adsorption, while it was shifted from 398.86 
$\mathrm{eV}$ to $399.52 \mathrm{eV}$ after Eu(III) adsorption, which illuminated that the nitrogen containing functional groups played roles not only as a cross-linker but also as adsorption sites for $\mathrm{Eu}(\mathrm{III})$ and $\mathrm{Au}$ (III). On the basis of characterization analysis as well as adsorption experiments, the mechanism of removing the targeted contaminant by DAWP-PEI- $\beta$-CD was schematically illustrated in Fig. 9. The mechanism of Eu(III) removal by DAWP-PEI- $\beta-C D$ was mainly through the surface coordination and host-guest inclusion interaction. Specifically, on the one hand, the ample amino groups of DAWP-PEI- $\beta$-CD that could form complex compounds with $\mathrm{Eu}(\mathrm{III})$. On the other hand, the cavities of $\beta-\mathrm{CD}$ could make contributions to the hostguest inclusion complexes with $\mathrm{Eu}$ (III). However, the adsorption mechanism of $\mathrm{Au}$ (III) on DAWP-PEI- $\beta-\mathrm{CD}$ was mainly attributed to the electrostatic attraction, chelation, host-guest inclusion, and redox reaction. Once the fresh DAWP-PEI- $\beta-C D$ was exposed to Au(III) solution, the targeted contaminants were rapidly enriched onto DAWP-PEI- $\beta$-CD surface via electrostatic interaction and followed the reduction of a part of $\mathrm{Au}(\mathrm{III})$ to $\mathrm{Au}(0)$ with the help of reductive functional groups. Finally, most of $\mathrm{Au}(\mathrm{III})$ and $\mathrm{Au}(0)$ were immobilized on the surface of DAWP-PEI- $\beta$-CD by the host-guest inclusion interaction.

\section{Conclusion}

In summary, a novel bio-adsorbent (DAWP-PEI- $\beta-C D$ ) was synthesized by grafting PEI and $\beta$-CD into dialdehyde waste paper via a facile two-step method. Characterization results found that the $\beta-C D$ and amino groups played an important role in uptake of $\mathrm{Eu}(\mathrm{III})$ and $\mathrm{Au}(\mathrm{III})$. Batch experiment showed that the isotherm and kinetic data were fitted well to the Langmuir and Pseudo-second-order models, and the saturated adsorption capacity of $\mathrm{Eu}(\mathrm{III})(\mathrm{pH}=6.0)$ and $\mathrm{Au}(\mathrm{III})(\mathrm{pH}=2.0)$ onto DAWP-PEI- $\beta$-CD were 424.2 and $241.3 \mathrm{mg} / \mathrm{g}$, respectively. The thermodynamic parameters suggested that the adsorption of $\mathrm{Eu}(\mathrm{III})$ and $\mathrm{Au}(\mathrm{III})$ on DAWP-PEI- $\beta$-CD were a spontaneous and endothermic processes. The adsorption mechanism study revealed that the elimination of Eu(III) was attributed to host-guest inclusion and surface complexation interactions, whereas adsorption of $\mathrm{Au}(\mathrm{III})$ might stem from a combination of electrostatic attraction, chelation, host-guest inclusion and oxidation-reduction interaction. Thus, these results will facilitate the development of new type biomass-based materials for $\mathrm{Eu}$ (III) and $\mathrm{Au}$ (III) pollution cleanup.

\section{Declarations}

\section{Acknowledgment}

We gratefully thank the Research Fund Program of National Natural Science Foundation of China (No. 21876115), Zhejiang Public Welfare Technology Application and Social Development Project (LGF21C030001).

\section{Author contributions}


Fenglei Liu, Shan Hua: Investigation, Data curation, Writing-original draft. Qingyuan Hu: Investigation, Project administration. Baowei Hu: Writing-review \& editing.

\section{Availability of data and material}

All data are available from the authors upon reasonable request.

\section{Conflict of interest}

There are no conflicts of interest do declare.

\section{References}

1. Al-Harahsheha M, AlJarraha M, Alrebakia M, Mayyasb M (2020) Nanoionic exchanger with unprecedented loading capacity of uranium. Sep Purif Technol 238:116423

2. Chen C, Hu J, Xu D, Tan X, Meng Y, Wang X (2008) Surface complexation modeling of Sr(II) and Eu(III) adsorption onto oxidized multiwall carbon nanotubes. J Colloid Interf Sci 323: 33-41

3. Chen X, Yang X, Xiang Y, Xu L, Liu G (2020) Humic acid derived resin: an efficient adsorbent for $\mathrm{Au}(\mathrm{III})$ uptake from aqueous acidic solution. J Chem Eng Data 65: 1824-1832

4. Cheng W, Wan T, Wang X, Wu W, Hu B (2018) Plasma-grafted polyamine/hydrotalcite as high efficient adsorbents for retention of uranium (VI) from aqueous solutions. Chem Eng J 324: 103111.

5. Fang Q, Duan S, Zhang J, Li J, Leung K (2017) Dual shelled $\mathrm{Fe}_{3} \mathrm{O}_{4}$ /polydopamine hollow microspheres as an effective Eu(III) adsorbent. J Mater Chem A 5: 2947-2958

6. Ghosh S, Badruddoza AZM, Uddin MS, Hidajat K (2011) Adsorption of chiral aromatic amino acids onto carboxymethyl-b-cyclodextrin bonded $\mathrm{Fe}_{3} \mathrm{O}_{4} / \mathrm{SiO}_{2}$ core-shell nanoparticles. J Colloid Interf Sci 354: 483-492

7. Guo Z, Li Y, Pan S, Xu J (2015) Fabrication of $\mathrm{Fe}_{3} \mathrm{O}_{4}$ @cyclodextrin magnetic composite for the highefficient removal of Eu(III). J Mol Liq 206: 272-277

8. Hu B, Wang H, Liu R, Qiu M (2021) Highly efficient U(VI) capture by amidoxime/carbon nitride composites: Evidence of EXAFS and modeling. Chemosphere. 274: 129743.

9. Huang S, Pang H, Li L, Jiang S, Wen T, Zhuang L, Hu B, Wang X (2018) Unexpected ultrafast and high adsorption of $\mathrm{U}(\mathrm{VI})$ and $\mathrm{Eu}(\mathrm{III})$ from solution using porous $\mathrm{Al}_{2} \mathrm{O}_{3}$ microspheres derived from MIL-53. Chem Eng J 353: 157-166.

10. Liu H, Zhou Y, Yang Y, Zou K, Wu R, Xia K, Xie S (2019) Synthesis of polyethylenimine/graphene oxide for the adsorption of $\mathrm{U}(\mathrm{VI})$ from aqueous solution. Appl Surf Sci 471: 88-95

11. Liu F, Wang S, Chen S (2020) Adsorption behavior of $\mathrm{Au}(\mathrm{III})$ and $\mathrm{Pd}(\mathrm{II})$ on persimmon tannin functionalized viscose fiber and the mechanism. Int J Biol Macromol 152: 1242-1251 
12. Liu F, Peng G, Li T, Yu G, Deng S (2019) Au(III) adsorption and reduction to gold particles on costeffective tannin acid immobilized dialdehyde corn starch. Chem Eng J 370: 228 -228 23

13. Liu F, Zhou Z, Li G (2021) Persimmon tannin functionalized polyacrylonitrile fiber for highly efficient and selective recovery of $\mathrm{Au}(\mathrm{III})$ from aqueous solution. Chemosphere 264: 128469

14. Liu F, Hua S, Zhou L, Hu B (2021) Development and characterization of chitosan functionalized dialdehyde viscose fiber for adsorption of $\mathrm{Au}(\mathrm{III})$ and Pd(II). Int J Biol Macromol 173: 457-466

15. Liu F, Zhou L, Wang W, Yu G, Deng S (2020) Adsorptive recovery of Au(III) from aqueous solution using crosslinked polyethyleneimine resins. Chemosphere 241: 125122

16. Liu F, Peng G, Li T, Yu G, Deng S (2019) Au(III) adsorption and reduction to gold particles on costeffective tannin acid immobilized dialdehyde corn starch. Chem Eng J 370: 228-236

17. Li S, Dong L, Wei Z, Sheng G, Hu B (2020) Adsorption and mechanistic study of the invasive plantderived biochar functionalized with CaAl-LDH for Eu(III) in water. J Environ Sci 96: 127-137.

18. Ma J, Zhao Q, Zhou L, Wen T, Wang J (2019) Mutual effects of U(VI) and Eu(III) immobilization on interpenetrating 3-dimensional $\mathrm{MnO}_{2}$ /graphene oxide composites. Sci Total Environ 695: 133696

19. Martin-Trasanco R, Cao R, Esparza-Ponce HE, Montero-Cabrera ME (2017) Reduction of Au(III) by a $\beta$ cyclodextrin polymer in acid medium, a stated unattainable reaction. Carbohyd Polym 175: 530-537

20. Neri G, Cordaro A, Cordaroa SM, Mazzaglia A, Piperno A (2021) PEGylated bis-adamantane carboxamide as guest bridge for graphene poly-cyclodextrin gold nanoassemblies. J Mol Struct 1240: 130519

21. Qiu M, Liu Z, Wang S, Hu B (2020) The photocatalytic reduction of U(VI) into U(IV) by ZIF-8/g- $C_{3} N_{4}$ composites at visible light. Environ Res 11: 110349.

22. Pestov A, Nazirov A, Modin E, Mironenko A, Bratskaya S (2015) Mechanism of Au(III) reduction by chitosan: Comprehensive study with ${ }^{13} \mathrm{C}$ and ${ }^{1} \mathrm{H}$ NMR analysis of chitosan degradation products. Carbohyd Polym 117: 70-77

23. Slavgorodska MV, Kyrychenko A (2020) Adsorption behavior of $\beta$-cyclodextrin onto gold nanoparticles. J Mol Graph Model 94: 107483

24. Shen H, Zhu G, Yu W, Wu H, Ji H, Shi H, She Y, Zheng Y (2015) Fast adsorption of p-nitrophenol from aqueous solution using $\beta$-cyclodextrin grafted silica gel. Appl Surf Sci 356: 1155-1167

25. Sun Y, Chen C, Tan X, Shao D, Li J, Zhao G, Yang S, Wang Q, Wang X (2012) Enhanced adsorption of $\mathrm{Eu}$ (III) on mesoporous $\mathrm{Al}_{2} \mathrm{O}_{3}$ /expanded graphite composites investigated by macroscopic and microscopic techniques. Dalton Trans 41: 13388-13394

26. Wang J, Wang Y, Wang W, Pen T, Liang J, Li P, Pan D, Fan Q, Wu W (2020) Visible light driven Ti ${ }^{3+}$ self-doped $\mathrm{TiO}_{2}$ for adsorption-photocatalysis of aqueous U(VI). Environmen Pollut 262: 114373

27. Wang H, Liu Y, Zeng G, Hu X, Hu X, Li T, Li H, Wang Y, Jiang L (2014) Grafting of $\beta$-cyclodextrin to magnetic graphene oxide via ethylenediamine and application for $\mathrm{Cr}(\mathrm{VI})$ removal. Carbohyd Polym 113: $166-173$ 
28. Wang F, Zhao J, Zhu M, Yu J, Hu Y, Liu H(2015) Selective adsorption-deposition of gold nanoparticles onto monodispersed hydrothermal carbon spherules: a reduction-deposition coupled mechanism. J Mater Chem A 3: 1666-1674.

29. Wang $M$, Cheng W, Wan T, Hu BW, Zhu YL, Song XF, Sun YB (2019) Mechanistic investigation of $U(V I)$ sequestration by zero-valent iron/activated carbon composites. Chem Eng J 362: 99-106.

30. Yan Z, Huang Q, Wang L, Zhang F (2019) Synthesis of tailored bis-succinamides and comparison of their extractability for U(VI), Th(IV) and Eu(III). Sep Purif Technol 213: 322-327

31. Yang S, Li Q, Chen L, Chen Z, Pu Z, Wang H, Yu S, Hu B, Chen J, Wang X (2019) Ultrahigh sorption and reduction of $\mathrm{Cr}(\mathrm{VI})$ by two novel core-shell composites combined with $\mathrm{Fe}_{3} \mathrm{O}_{4}$ and $\mathrm{MoS}_{2}$. $\mathrm{J} \mathrm{Hazard}$ Mater 379: 120797.

32. Zhao F, Repo E, Yin D, Meng Y, Jafari S, Sillanpaä M (2015) EDTA-cross-linked ßcyclodextrin: an environmentally friendly bifunctional adsorbent for simultaneous adsorption of metals and cationic dyes. Environ Sci Technol 49: 10570-10580

33. Zhao B, Yuan L, Wang Y, Duan T, Shi W (2021) Carboxylated UiO-66 tailored for U(VI) and Eu(III) trapping: from batch adsorption to dynamic column separation. ACS Appl Mater Interfaces 13: $16300-16308$

34. Zhang Q, Zhao D, Feng S, Wang Y, Jin J, Alsaedi A, Hayat T, Chen C (2019) Synthesis of nanoscale zero-valent iron loaded chitosan forsynergistically enhanced removal of $\mathrm{U}(\mathrm{VI})$ based on adsorption andreduction. J Colloid Interf Sci 552: 735-743

35. Zhu Y, Wang X, Li Z, Fan Y, Zhang X, Chen J, Zhang Y, Dong C, Zhu Y (2021) Husbandry waste derived coralline-like composite biomass material for efficient heavy metal ions removal. Bioresource Technol 337: 125408

36. Zhang M, Dong Z, Hao F, Xie K, Qi W, Zhai M, Zhao L (2021) Ultrahigh and selective adsorption of $\mathrm{Au}(\mathrm{III})$ by rich sulfur and nitrogen-bearing cellulose microspheres and their applications in gold recovery from gold slag leaching solution. Sep Purif Technol 274: 119016

37. Zhao F, Repo E, Meng Y, Wang X, Yin D, Sillanpää M (2016) An EDTA-b-cyclodextrin material for the adsorption of rare earth elements and its application in preconcentration of rare earth elements in seawater. J Colloid Interf Sci 465: 215-224

38. Zhao F, Repo E, Song Y, Yin D, Hammouda SB, Chen L, Kalliola S, Tang J, Tam KC, Sillanpää M (2017) Polyethylenimine-cross-linked cellulose nanocrystals for highly efficient recovery of rare earth elements from water and a mechanism study. Green Chem 19: 4816-4828

\section{Figures}




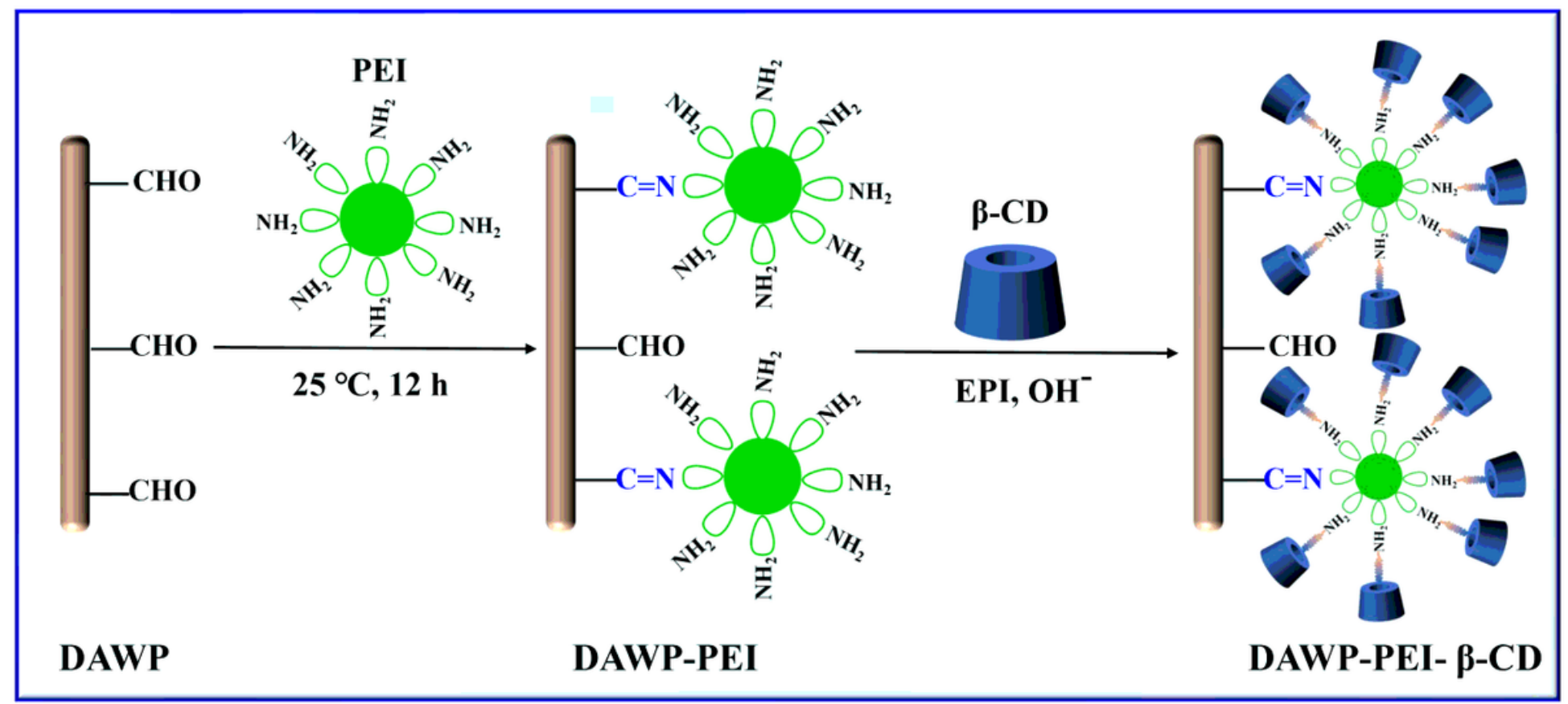

Figure 1

Schematic diagrams for the synthetic procedures of DAWP-PEI- $\beta-C D$. 


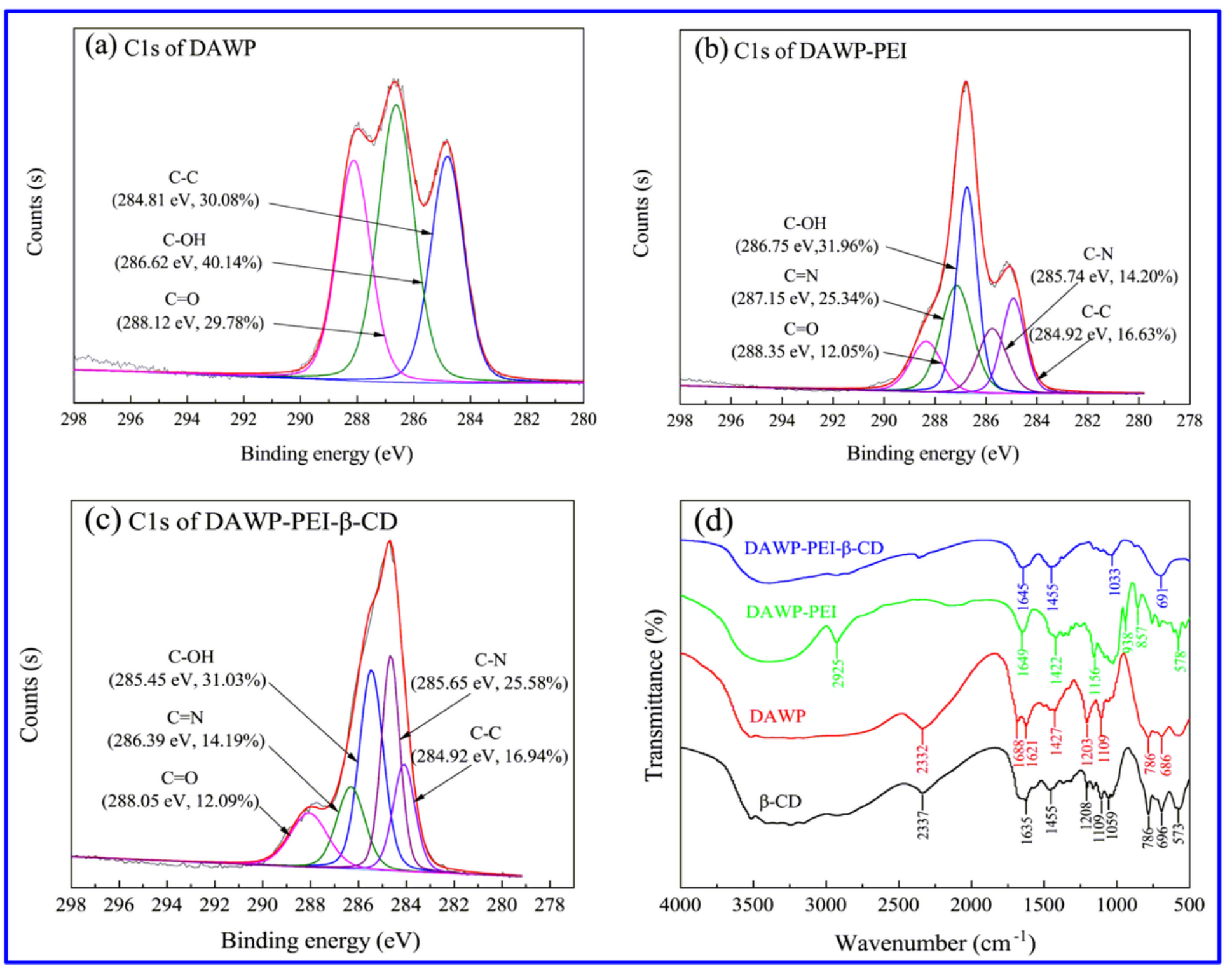

Figure 2

XPS C1s spectrum $(a, b, c)$ and FT-IR (d) of $\beta-C D$, DAWP, DAWP-PEI and DAWP-PEI- $\beta-C D$. 

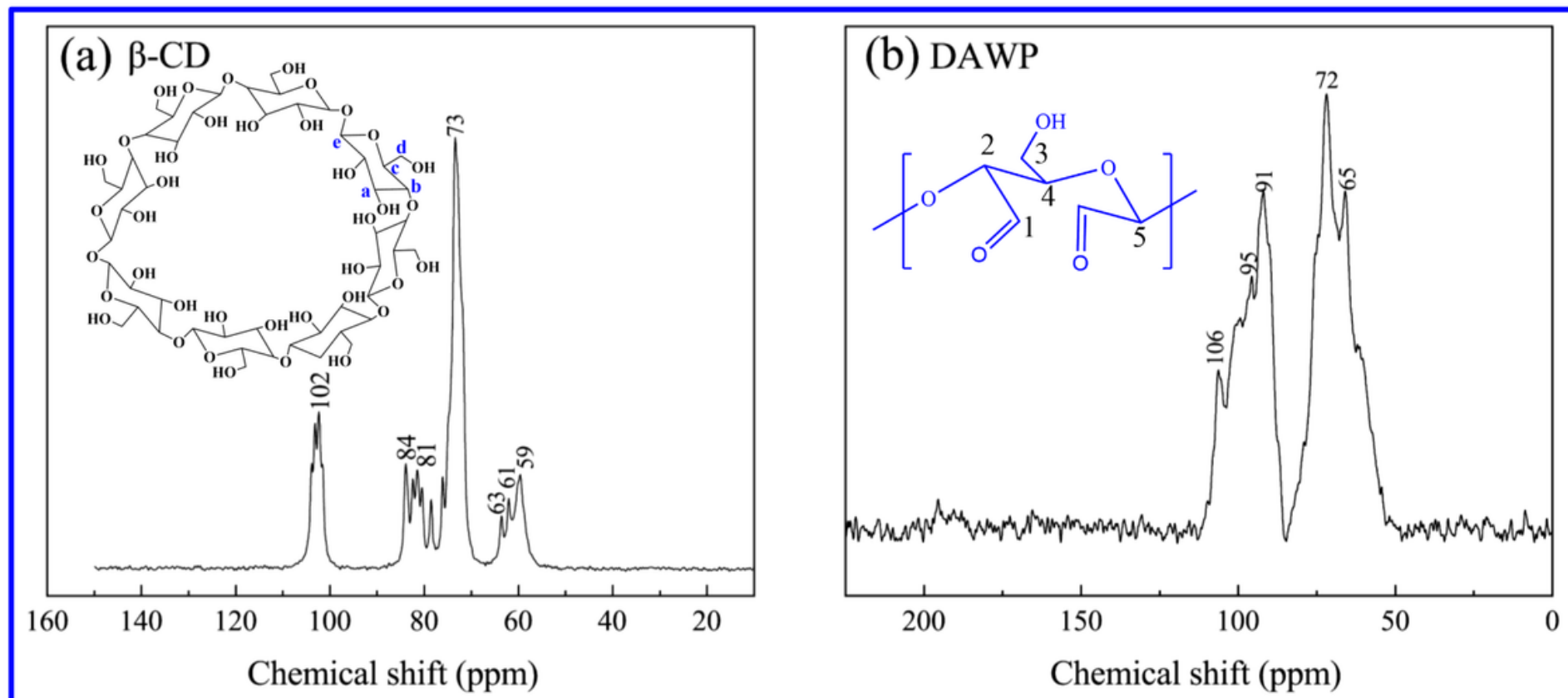

\section{(c) DAWP-PEI}

(d) DAWP-PEI- $\beta$-CD
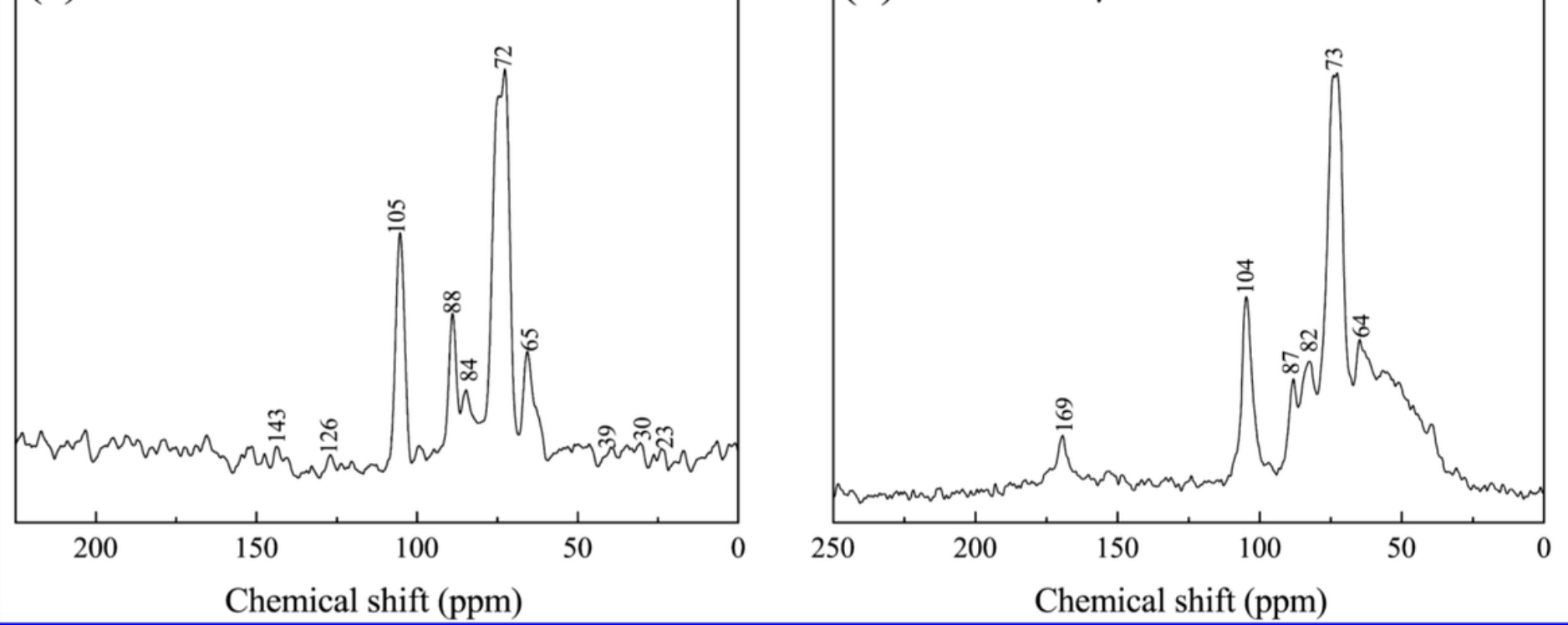

\section{Figure 3}

13C NMR spectra of $\beta-C D$, DAWP, DAWP-PEI and DAWP-PEI- $\beta-C D$. 


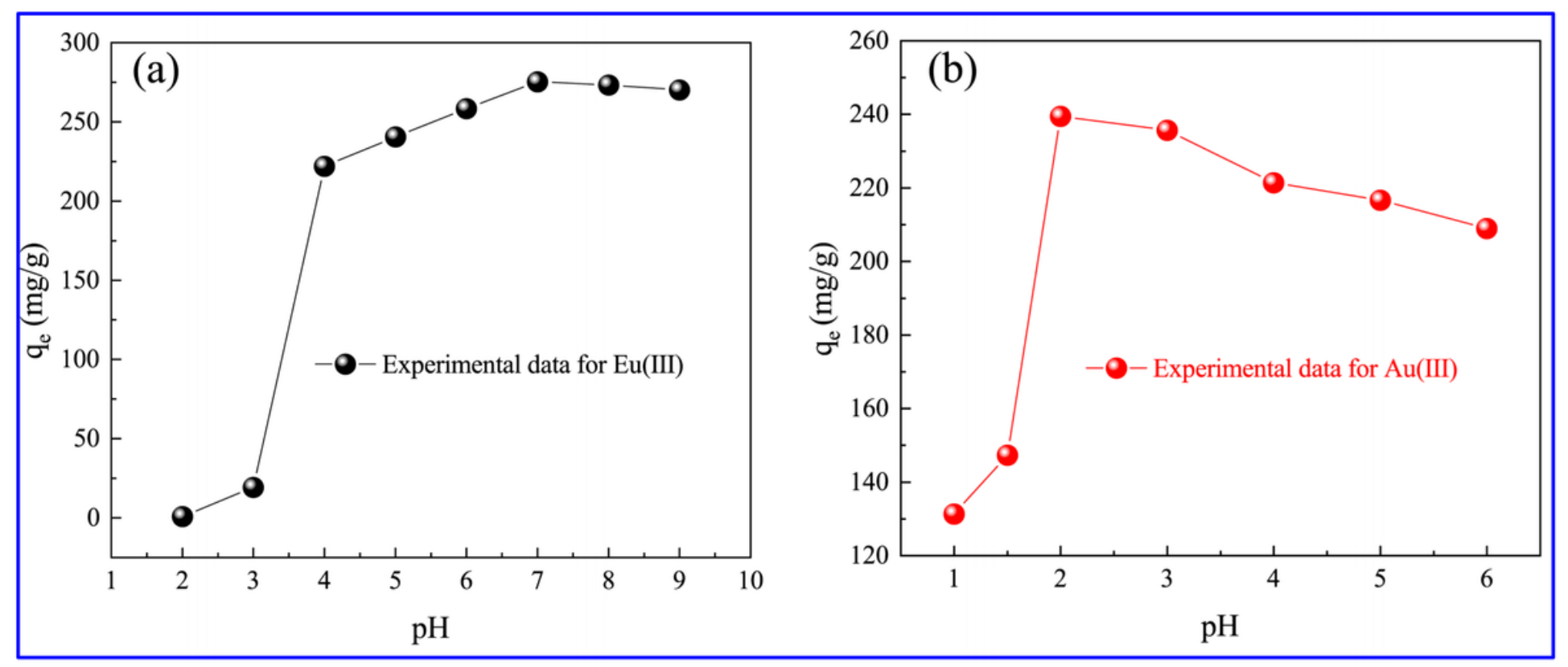

Figure 4

Effect of initial pH on elimination of Eu(III) and Au(III) by DAWP-PEI- $\beta$-CD. 


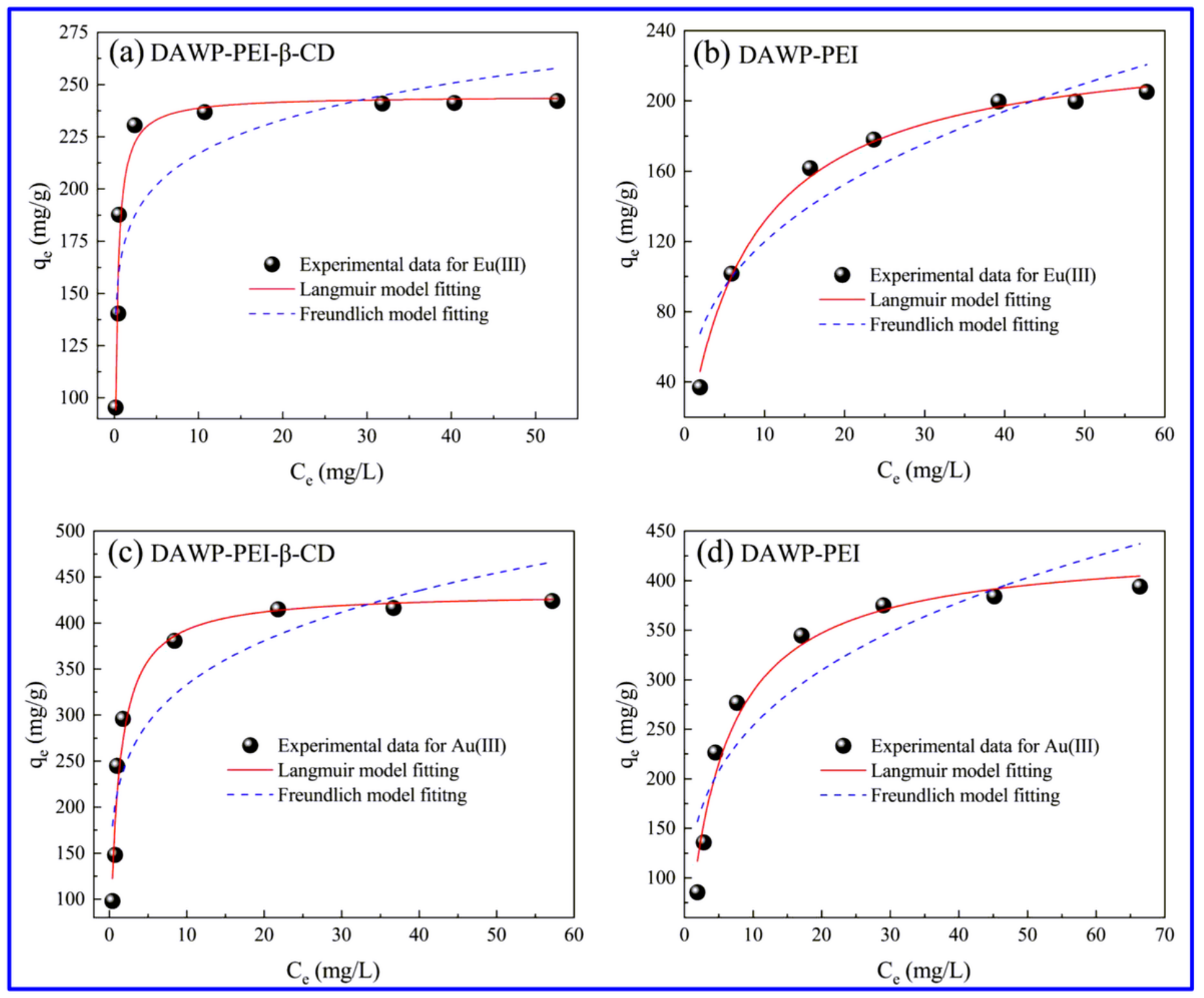

Figure 5

Sorption isotherms of Eu(III) and Au(III) on DAWP-PEI and DAWP-PEI- $\beta$-CD as well as fitting by the Langmuir and Freundlich equations. 


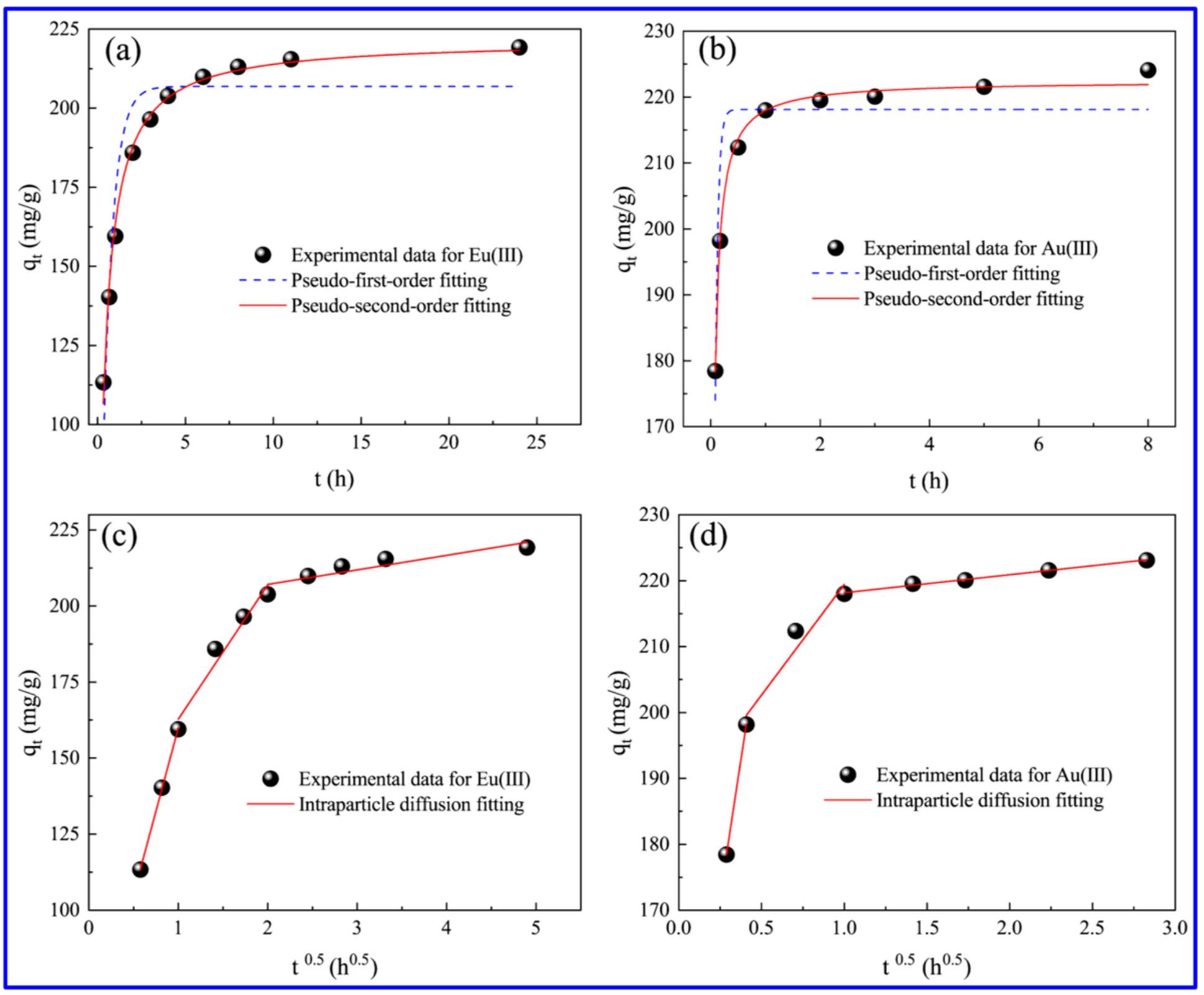

Figure 6

Effects of the equilibrium time on Eu(III) and Au(III) adsorbed on DAWP-PEI- $\beta$-CD as well as modeling by different kinetics equations. 


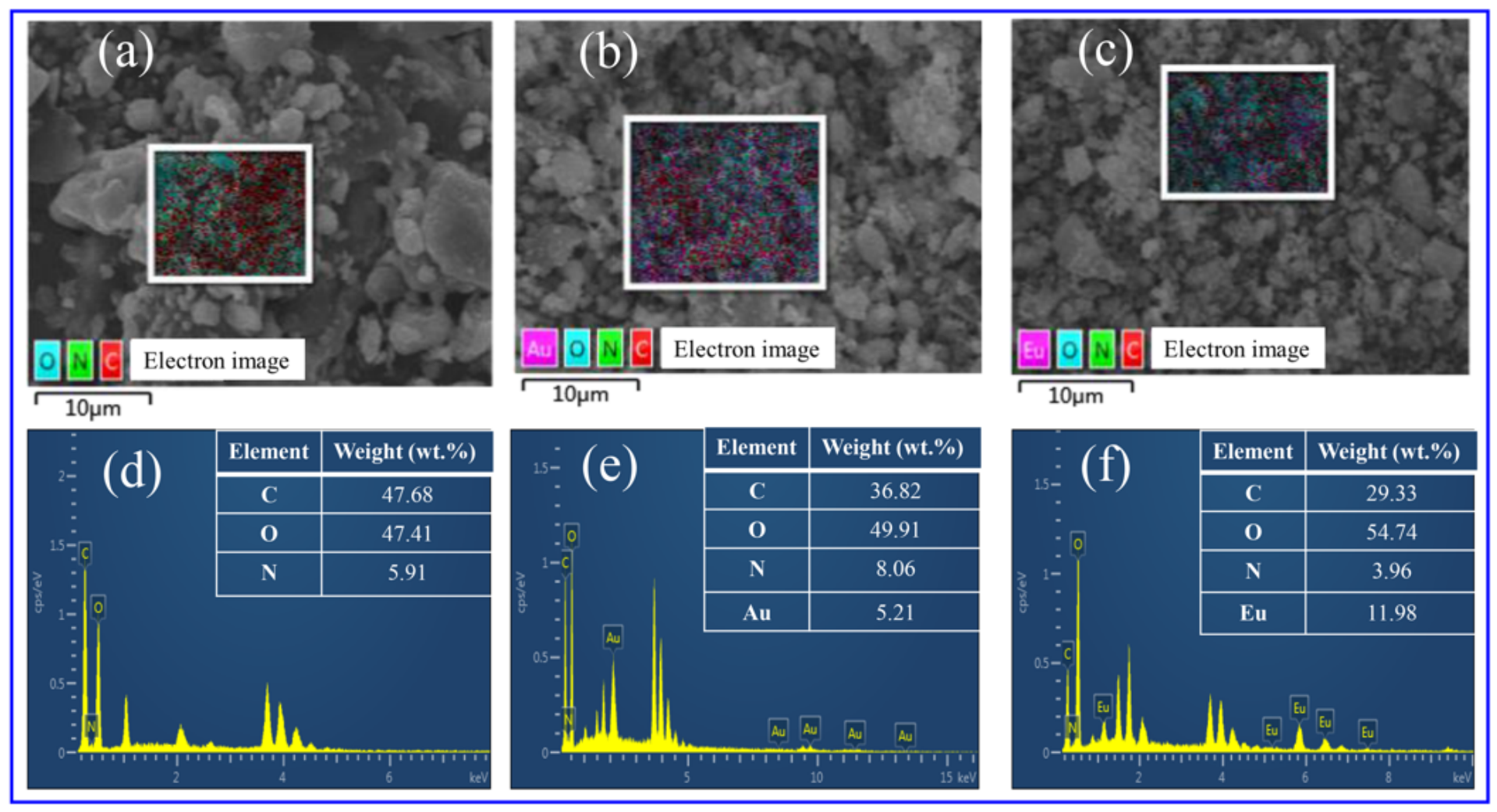

\section{Figure 7}

The SEM images and EDS spectrum of DAWP-PEI- $\beta$-CD ( $a, d)$, DAWP-PEI- $\beta$-CD-Au (b, e), DAWP-PEI- $\beta$-CD$\mathrm{Eu}(\mathrm{c}, \mathrm{f})$. 

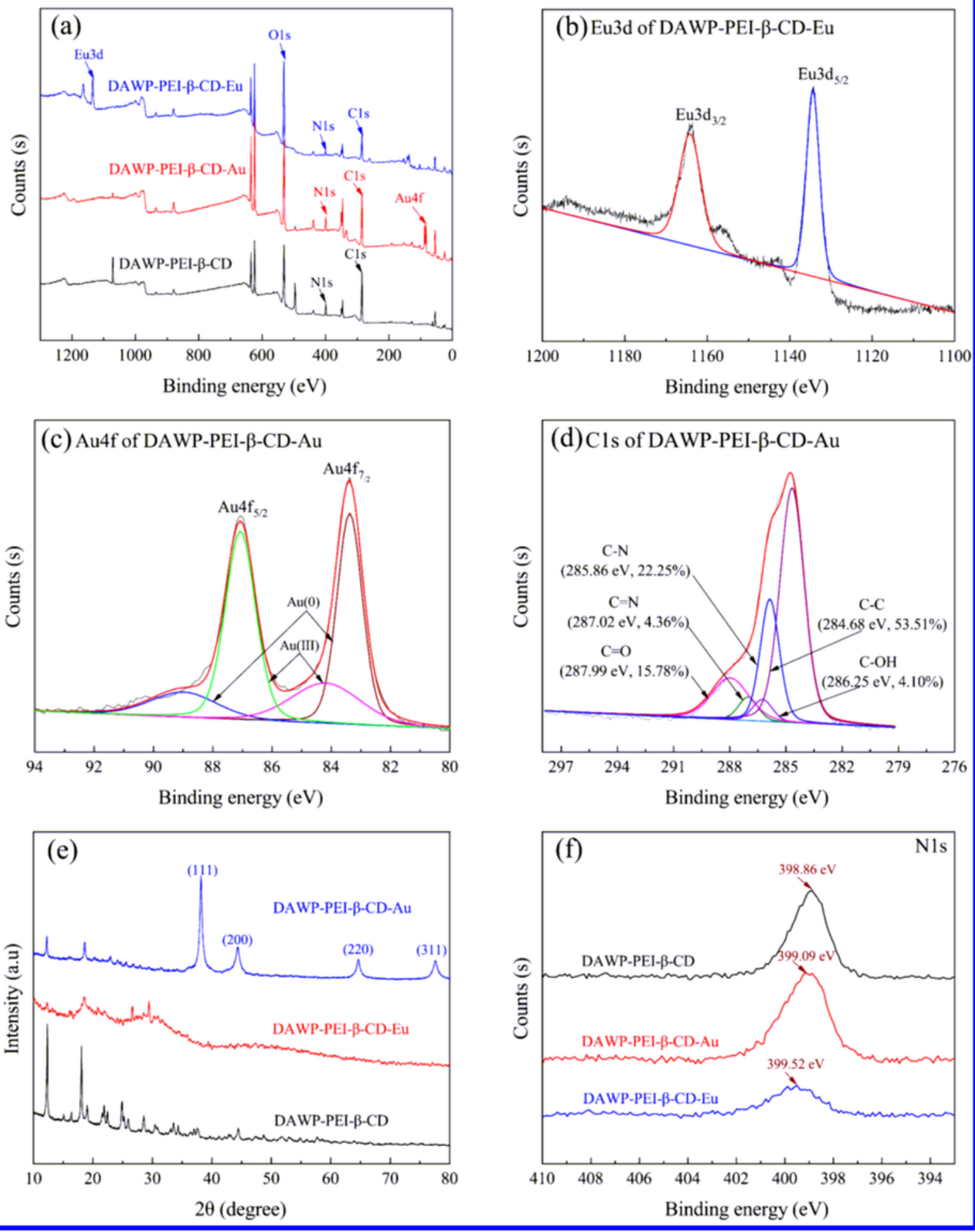

Figure 8

XPS survey (a), Eu3d, Au4f, C1s, N1s spectra (b, c, f) and XRD patterns (e) of DAWP-PEI- $\beta$-CD, DAWP-PEI- $\beta$ CD-Au, DAWP-PEI- $\beta$-CD-Eu. 


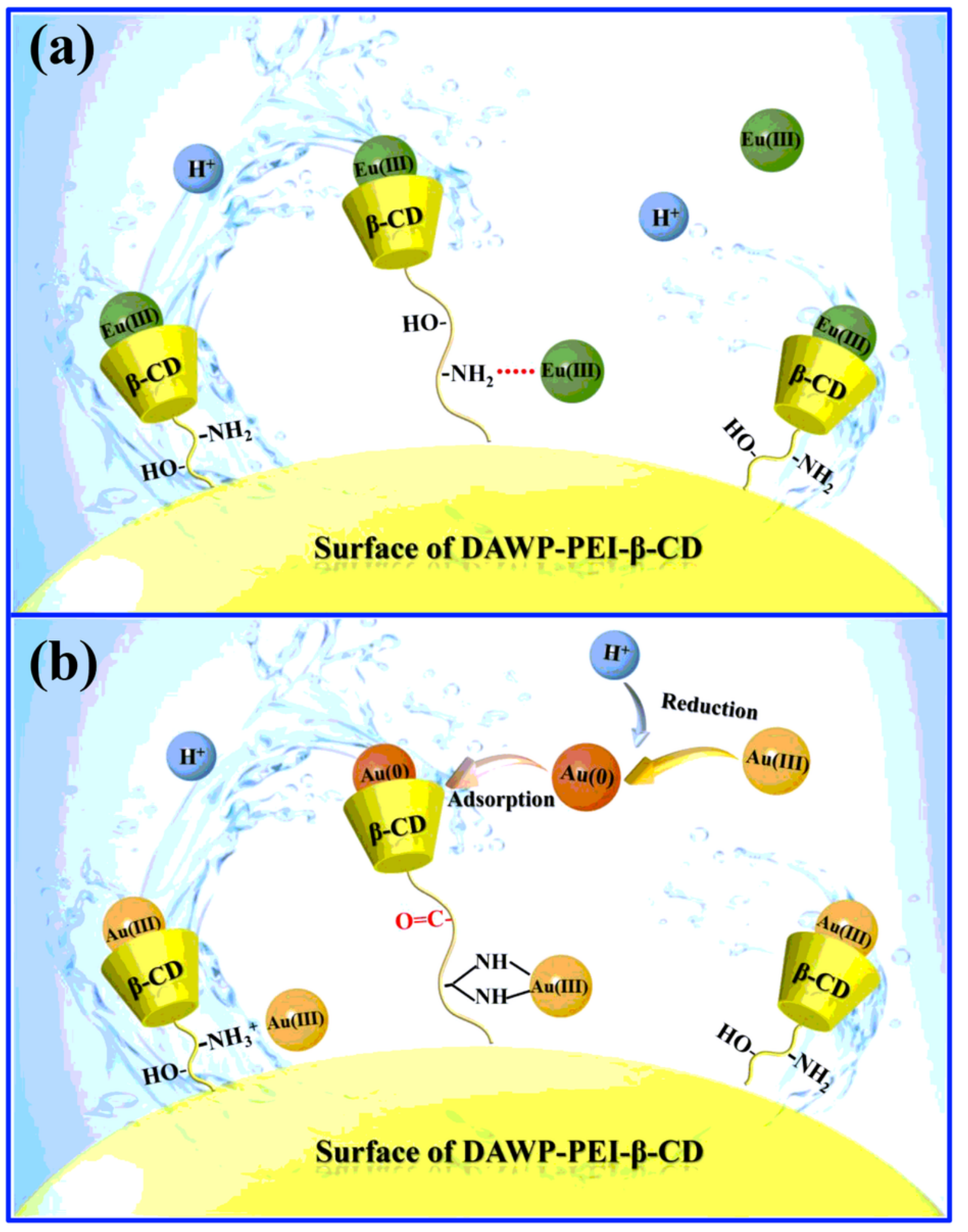

Figure 9

Schematic diagram of the plausible adsorption mechanism of Eu(III) and Au(III) by DAWP-PEI- $\beta-C D$.

\section{Supplementary Files}

This is a list of supplementary files associated with this preprint. Click to download. 
- GraphicalAbstract.png

- SupportingMaterials.docx 